\title{
Data Needs for a Serious IIIness Care Accountability System: A Framework and Recommendations
}

\author{
Tom Valuck, MD, JD and Russ Montgomery, PhD, MHS
}

\begin{abstract}
Background: Successful implementation of a comprehensive accountability system for community-based serious illness care will require a robust data infrastructure. Data will be needed to support care delivery, quality measurement, value-based payment, and evaluation and monitoring.

Objective: The specific data needs in these areas need to be identified and understood, so that gaps in currently available data may be addressed.

Design: We developed a framework that includes the needed data and data infrastructure to support the features and characteristics of a serious illness care accountability system. Based on this framework, we analyze the current data landscape to identify gaps in available data resources and capacities. This analysis was informed by conducting Internet-based research, interviews with key informants, and a survey of key informants.

Results: Based on the identified gaps, we present a series of priority recommendations for advancing the data infrastructure to support community-based serious illness care. These recommendations include additional measurement of patient-reported outcomes, increasing interoperability among various data sources, increasing development and exchange of patient care plans, leveraging newly standardized data on patient functional and cognitive status, and using patient-reported information for clinical decision support at the point of care.

Conclusion: There are significant unmet data needs for a comprehensive accountability system in serious illness care, but these gaps can be prioritized and addressed through alignment and collaboration across stakeholders.
\end{abstract}

Keywords: accountability system; care delivery; community-based care; quality measurement; serious illness; value-based payment

\section{Introduction}

$\mathbf{R}$ OBUST DATA are needed for multiple purposes in an accountability system for community-based serious illness care. These purposes include care delivery, quality measurement, value-based payment, and evaluation and monitoring. However, data are often poorly aligned and inaccessible when needed due to fragmentation in our healthcare system. Such fragmentation is particularly severe in serious illness care. In other cases, data are not being generated. It is necessary to identify existing gaps in needed data and ways to fill these gaps.
In this article, we present a framework of the data needs for an accountability system and highlight the gaps in currently available data. We then present a series of recommendations on filling data gaps.

\section{Methods}

We conducted an analysis to identify data needs based on a set of design characteristics for a serious illness care accountability system, which builds on the introductory article of Teno et al. in this special issue. This analysis resulted in a framework that presents design characteristics and data

Discern Health, Baltimore, Maryland.

Accepted October 27, 2017.

This article was developed for, and discussed at, a meeting titled "A Convening on Quality Measures for Serious Illness Care," which was held May 9-11, 2017 in Banff, Canada. The meeting was organized by the Gordon and Betty Moore Foundation, the Cambia Palliative Care Center of Excellence at the University of Washington, the Center to Advance Palliative Care, and the Icahn School of Medicine at Mount Sinai. This supplement is funded by the Gordon and Betty Moore Foundation. 


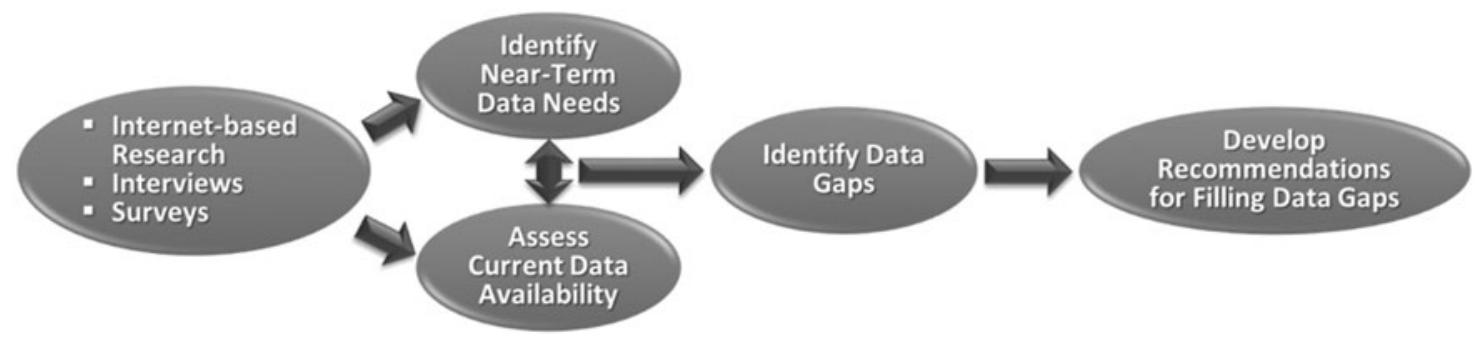

FIG. 1. Analytic approach.

needs, along with data types and sources that may be used to meet these needs. In addition, we conducted an analysis to assess the extent to which existing data meet the needs described in the framework. The analyses were informed by Internet-based research, interviews with key informants, and a survey of a convenience sample of key informants. Figure 1 summarizes the analytic approach.

\section{Data needs framework}

Figure 2 is a framework that highlights a set of defining characteristics for a robust accountability system for high quality, affordable, community-based serious illness care. These characteristics are divided across four domains:

1. Care delivery,

2. Quality measurement,

3. Value-based payment, and

4. Monitoring and evaluation.

Based on these characteristics, the framework presents a set of data needs for each of the four domains. These data needs are divided into data processes and end uses of data. In addition, the framework identifies the major types of data and major available data sources that may be used to meet the defined needs.

This framework does not represent the ideal future, but rather what may be possible in the near- to midterm. Below, we review the data sources and types in the framework, and then map them to the identified data needs within each domain. This mapping of data types and sources includes data that are currently being used to meet the defined needs or have the potential to be leveraged in the near- to midterm to meet the needs.

\section{Data sources and types}

A range of data sources can be leveraged for care delivery, quality measurement, value-based payment, and monitoring and evaluation. Claims databases aggregate utilization and cost data from payers and purchasers. Electronic health records (EHRs) and paper medical records contain patient-level clinical data, such as diagnoses, histories, symptoms, test results, and medications. In rare cases, they include data on patient functional and cognitive status.

Patients and their families are data sources through surveys, which may be used at the provider-level for quality improvement and aggregated in registries and other repositories for quality measurement, payment, and other uses. Patient-reported outcomes (PROs) focus on outcomes such as symptom management and patient experience, among other types of data. Notably, when a seriously ill patient serves as a data source, steps must be taken to reduce reporting burden. Proxies may be used to alleviate the reporting burden. While studies show that responses from patients and their

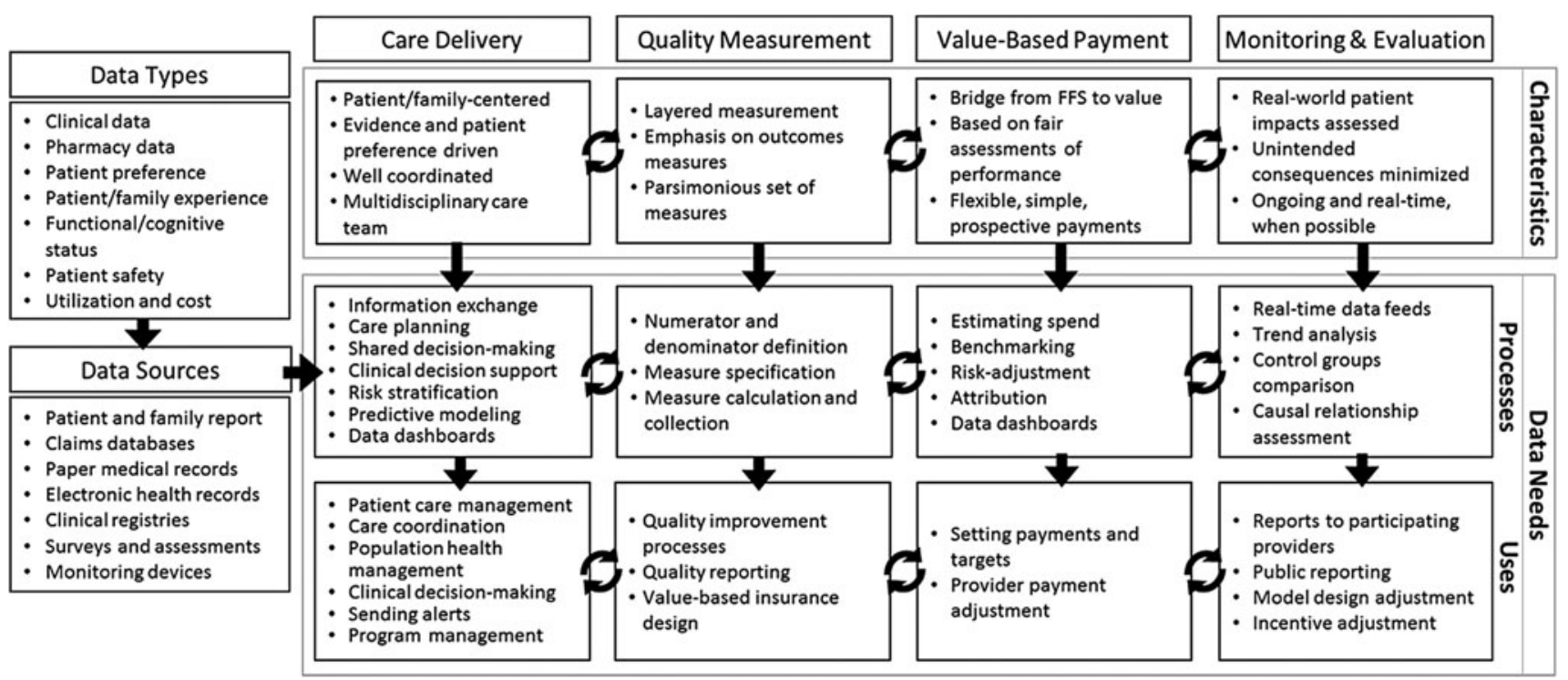

FIG. 2. Data needs framework for a serious illness care accountability system. 
proxies often differ, particularly on subjective symptoms, ${ }^{1}$ a family member is often the best source of information for some seriously ill patients (e.g., individuals with end-stage dementia). $^{2}$

Patient assessments include data elements such as functional and cognitive status and patient and family experience, as well as clinical data. To serve as a data source for population health management and other uses, assessments may be aggregated and reported at a population-level. Similarly, clinical registries aggregate a wide range of data at a population-level, including clinical data and functional and cognitive status. Monitoring devices are an emerging source of patient-level data. They may report clinical data such as vital signs or may track functional status and cognition, among other types of data.

\section{Care delivery}

Characteristics. Studies show that seriously ill individuals prefer to receive care at home, rather than in institutional settings. ${ }^{3}$ The seriously ill have complex needsboth medical and nonmedical. To most effectively treat their conditions and provide care that is patient-centered, service delivery should be evidence-based and driven by patient preferences. The seriously ill benefit from care that is multidisciplinary, team-based, and well-coordinated across care settings.

Data needs. The data types in the framework are used in a variety of ways to support care delivery. At the patientlevel, clinical data are used to manage and coordinate care and support clinical decision making. Clinical decision support helps providers make informed decisions at the point of care, and care planning and shared decision-making help ensure that these decisions align with patient preferences. At the population-level, data dashboards present aggregated clinical data as well as other types of data, such as functional and cognitive status, health outcomes, and utilization and cost, to more effectively manage population health and engage in program and system management. Within dashboards and other data tools, risk stratification and predictive modeling may be used to identify and segment patients by acuity or other factors to more effectively target interventions.

\section{Quality measurement}

Characteristics. Effective quality measurement links structure and process measures to outcome measures in accordance with the Donabedian framework. ${ }^{4}$ Outcome measures, particularly PROs, should be emphasized. Measure sets should include reliable and valid measures of the factors important to quality care and outcomes for the seriously ill. In addition, measure sets should be parsimonious, with common measures used across care settings and programs where possible, and additional measures specific to the setting and purpose used as needed. The collection and reporting of measures should minimize burden on the provider, patient, and family.

Data needs. Nearly each type of data can and should be used for quality measurement. For the serious illness population, PROs and data on patient preference and patient and family experience are particularly important. In addition, quality measures must be specified, including clear numerator and denominator definitions. This requires rich data on characteristics that define the serious illness population, such as diagnosis as well as functional and cognitive status. Once calculated, measures may be used for a variety of purposes, including public reporting, value-based payment, and quality improvement. In addition, performance information may also be used for value-based insurance design by establishing provider networks and cost-sharing adjustments that encourage beneficiaries to choose high-quality providers.

\section{Value-based payment}

Characteristics. Payment should move from fee-forservice, based on the volume of services provided, to the value of care delivered. To support value-based care, provider payment should be tied to performance, with steps taken to ensure fair and timely comparisons across providers. To maximize the ability of clinicians to provide the care needed by the seriously ill when they need it, payment should be flexible and prospective. Simple payment structures help minimize provider uncertainty and administrative burden.

Data needs. Historical information on utilization, cost, and quality measure performance are needed to structure value-based payment models and set performance targets. To help ensure fair comparisons and make payment adjustments, measures should be benchmarked and risk-adjusted. Benchmarking allows for comparisons across providers and against targets and trends, while risk-adjustment helps ensure that measures are based on performance and not on other differences in the patient population. Once measures are risk-adjusted and benchmarked, they can be used to adjust payment.

\section{Monitoring and evaluation}

Characteristics. Short-term monitoring and long-term evaluation are needed to assess the real-world impacts of healthcare delivery and payment policy changes on patients. Monitoring and evaluation should focus on the effectiveness of care and payment models, as well as potential unintended consequences, such as restricted patient access to services and technologies. These processes should be ongoing from the initiation of new models.

Data needs. For monitoring purposes, real-time data feeds (or close to real-time) are needed to identify issues and address them promptly. For evaluations, data are needed to identify trends, conduct studies that compare treatment and comparison groups, and assess causal relationships between program features and outcomes, including impacts on utilization and cost. The results of these evaluations should be reported to participating providers, who may use them for quality improvement; policymakers, who may use them to improve programs; and patients, who may use them to make more informed care decisions. In addition, results may be used to adjust the design of care models and the incentives that are part of payment models. 


\section{Data Gaps and Recommendations}

We assessed the extent to which currently available data are meeting the needs described in the framework. Based on this assessment, we identified a series of data gaps and recommendations for filling these gaps. The gaps and recommendations have been grouped together into broad goals for improving data availability and the data infrastructure to support an accountability system.

\section{Goal 1: Increase patient- and family-centered care}

Providing care that is patient- and family centered is an aim of the National Quality Strategy and has been a goal in the ongoing evolution toward value-based payment. ${ }^{5}$ Capturing patient preferences and perspectives on care is even more essential in serious illness care, as patients may not be expected to recover and the goals of care are oriented toward managing symptoms and improving quality of life.

Recommendation: Increase aggregation of PROs and develop PRO-performance measures. The collection of true PROs is currently limited in serious illness care, and aggregation of PROs for the development and use of PROperformance measures is minimal. As new clinical registry capabilities are planned, there is an opportunity to include additional collection and aggregation of PROs. By making registry participation a requirement for participating in an accountability system, small numbers issues can be mitigated, and incentive models can take into account the extent to which providers are addressing patient preferences and experiences.

Recommendation: Increase collection and exchange of longitudinal care plans and advance directives. True patient- and family-centered care is not possible if providers do not have access to detailed patient care plans and information on patient preferences for care. Unfortunately, these documents are often not developed during care. When they are developed, they are often not shared and are not available to clinicians when they are needed. Initiatives to increase collection of advance directives and other end-of-life care orders, such as physician orders for life-sustaining treatment (POLST), have been implemented in recent years, largely at the state level.

As of 2017, 48 states have POLST programs, although actual submission of documents to them remains low, and the adequacy of communication during care planning has been questioned. ${ }^{6,7}$ There is an opportunity to build engagement with these programs and help them expand and improve. While these initiatives may increase development of advance directives, the documents are often not made accessible via EHRs. Working with EHR vendors may help address some of the accessibility issues. Importantly, POLST should be reviewed and updated regularly to ensure that they continue to capture patient preferences.

\section{Goal 2: Reduce data collection and reporting burden}

A major challenge in collecting data about seriously illness is the burden placed on patients, families, and providers. This is true for data entry in EHRs, conducting patient assessments in postacute care settings, data entry into clinical registries, and gathering patient-reported information. Many of the data elements are similar or redundant across these collection methods and data resources.

Recommendation: Increase data exchange and interoperability. While strides have been made in recent years, data exchange and interoperability across care settings remain low and inadequate for quality improvement and other uses. As part of IMPACT Act implementation, The Centers for Medicare and Medicaid Services (CMS) is developing a Data Elements Library (DEL) to facilitate interoperability and transferability of data from postacute care data sets. ${ }^{8}$ Through the DEL, CMS is mapping the data elements from patient assessments to the Consolidated Clinical Document Architecture, which is a widely used standard that defines many of the types of information built into EHRs. Providers can use the information in the DEL to help establish interoperability for their EHRs.

With the DEL and growing interoperability across care settings, there are new opportunities to exchange data to populate EHRs, assessments, and other data sources. Such data exchange can reduce the frequency of repetitive data collection. Reporting requirements for any new accountability system should harness these advancements in standards for data exchange and interoperability to reduce burden.

Recommendation: Identify measurement approaches that minimize patient and family burden. Increased interoperability between assessments, EHRs, and other data sources may also reduce the reporting frequency for patients and families. However, even with decreased frequency, patients and their families will still face reporting burden. Future research on ways to address several critical measurement issues with PROs can help to address many of these issues. These topics include ways to improve the validity of proxy use, provide questionnaires that are more appropriate to differing levels of patient function (e.g., vision and cognition), reduce instances of false representation of symptoms by providers and patients, and elicit patient responses using passive technologies.

\section{Goal 3: Improve EHR functionality}

Enhanced data exchange and interoperability with EHRs will only advance so far without changes to EHR functionality and interfaces. While standards and language have advanced in some ways, many EHR platforms simply do not have fields or displays for certain types of information, such as care plans and advance directives.

Recommendation: Work with partners to propose enhanced functions and codes to EHR vendors. Multiple key informants stated that the terminology used in hospice care and other settings relevant to serious illness is not as well represented in the Logical Observation Identifiers Names and Codes (LOINC), which is a set of code names and identifiers for medical terminology used in EHRs. EHR vendors may be willing to speak with providers on these issues, but without a critical mass of EHR users requesting changes, vendors are unlikely to make significant modifications. There is an opportunity to organize specialty societies and provider trade associations to approach vendors in a unified manner with concrete requests. Such an approach could also help by 
spreading the costs of changes across many different consumers.

As a complementary strategy, a consortium of these organizations could also work to develop lists of priority terms for serious illness and approach the Regenstrief Institute, the organization that oversees LOINC, to request development of additional codes. These codes would permit increased processing of care plans and other open text in EHRs for quality improvement, measurement, and other purposes.

\section{Goal 4: Define and segment the serious illness population}

Defining the serious illness population and leveraging data related to that definition is necessary for all four domains of data needs. In particular, data on physical and cognitive function are needed to segment the population and define and calculate denominators for quality measures. Moreover, defining and segmenting the population can help to determine which patients should be included in new models of care, as well as value-based payment models and program evaluations.

Recommendation: Leverage functional and cognitive status data in postacute care datasets and clinical registries. Functional and cognitive status information is included in the recommended quality measure definitions in the article of Kelley in this special issue. The implementation of the IMPACT Act is bringing much needed standardization to functional status data elements in the postacute care datasets, including the Minimum Data Set, Inpatient Rehabilitation Facility-Patient Assessment Instrument, Outcome and Assessment Information Set, and Long-Term Care Hospital CARE Data Set. ${ }^{9}$ Cognition items are also being standardized, including mental status assessment tools such as the Brief Interview on Mental Status. These data can be leveraged to define the serious illness population and calculate measure denominators, as described in the article of Kelley.

\section{Goal 5: Improve accessibility of data at the point of care}

Clinical decision support can lead to more informed decisions at the point of care. However, due to the need for data from multiple settings of care, clinical decision support in serious illness care has not been as advanced as other areas of healthcare. New technology and the emergence of big data and new data sources are resulting in new capabilities for improving clinical decision support.

Recommendation: Build "feed forward" capability into clinical data registries for clinical decision support. The clinical data registries currently in use in serious illness care, including the Global Palliative Care Quality Alliance, ${ }^{10}$ Palliative Care Quality Network, ${ }^{11}$ and National Palliative Care Registry, ${ }^{12}$ are used primarily for local quality improvement and research. They do not have built-in capability to facilitate real-time clinical decision support based on PROs.

A planning process is currently underway to enhance and integrate the existing registries, and there is an opportunity to add "feed forward" capabilities to help inform decision making at the point of care. This approach was pioneered by the Swedish Rheumatology Quality registry. Patients enter, or feed forward, information about their symptoms and quality of life, through a web portal or on registration during an office visit. The provider supplements this information with data from a clinical examination and laboratory tests. A built-in decision support system incorporates these data and shows comparisons to the data of other patients in the registry. ${ }^{13}$

\section{Conclusion}

Based on our review of the current landscape, additional data and data capabilities are needed to support serious illness care delivery, quality measurement, value-based payment, and evaluation and monitoring. To fill gaps in currently available data, we recommend actions to achieve specific goals.

To support care delivery, EHRs and clinical data registries need additional functionality to inform clinical decision making, and increased use of PROs and advanced care plans will help ensure patient preferences are informing care. To support quality measurement, payment, and evaluation and monitoring, the serious illness population needs to be better defined and segmented. As new data and data infrastructure are developed, efforts should be made to reduce the data reporting burdens on patients, families, and providers. Addressing these recommendations will require new ways of thinking and collaborating among organizations.

\section{Acknowledgment}

This work was funded by the Gordon and Betty Moore Foundation.

\section{Author Disclosure Statement}

No competing financial interests exist.

\section{References}

1. Li M, Harris I, Lu ZK: Differences in proxy-reported and patient-reported outcomes in assessing health and functional status among Medicare beneficiaries. BMC Med Res Methodol 2015;15:62.

2. Teno, JM, Clarridge BR, Casey V, et al.: Family perspectives on end-of-life care at the last place of care. JAMA 2004;291:88-93.

3. AARP: Aging in Place: A State Survey of Livability Policies and Practices. http://assets.aarp.org/rgcenter/ppi/liv-com/agingin-place-2011-full.pdf. (Last accessed August 19, 2017).

4. Donabedian A: The quality of care: How can it be assessed? JAMA 1988;260:1743-1748.

5. Agency for Healthcare Research and Quality: 2015 National Healthcare Quality and Disparities Report and 5th Anniversary Update on the National Quality Strategy. www.ahrq. gov/sites/default/files/wysiwyg/research/findings/nhqrdr/ nhqdr15/2015nhqdr.pdf. (Last accessed September 29, 2017).

6. National POLST Paradigm: POLST in Your State. http://polst. org/programs-in-your-state. (Last accessed August 18, 2017).

7. Moore KA, Rubin EB, Halpern SD: The problems with physician orders for life-sustaining treatment. JAMA 2016; 315:259-260.

8. CMS Medicare Learning Network: The IMPACT Act and the Data Element Library. www.cms.gov/Medicare/ 
Quality-Initiatives-Patient-Assessment-Instruments/PostAcute-Care-Quality-Initiatives/Downloads/April-14-2016-MLNConnects-NPC-IMPACT-Act-and-Data-Element-Library.pdf. (Last accessed September 14, 2017).

9. CMS Medicare Learning Network: The IMPACT Act of 2014 and Data Standardization. www.cms.gov/Outreachand-Education/Outreach/NPC/Downloads/2015-10-21-PostAcute-Care-Presentation.pdf. (Last accessed October 9, 2017).

10. Global Palliative Care Quality Alliance: www.gpcqa.org. (Last accessed September 13, 2017).

11. Palliative Care Quality Network: QDACT. www.gpcqa. org/qdact. (Last accessed September 13, 2017).
12. National Palliative Care Registry: Registry Information. https://registry.capc.org/registry-information. (Last accessed September 13, 2017).

13. Swedish Rheumatology Quality Register: About SRQ http://srq.nu/en/about-srq. (Last accessed August 14, 2017).

Address correspondence to: Russ Montgomery, PhD, MHS

Discern Health

1120 N. Charles Street, Suite 200

Baltimore, MD 21201

E-mail: rmontgomery@discernhealth.com 